\title{
Estrategias de comunicación para crear alianzas
}

\author{
a) \\ Ana María Suárez Monsalve* \\ Recibido: 19 de abril de 2009 \\ Aprobado: 06 de mayo de 2009
}

\begin{abstract}
RESUMEN
Este artículo de reflexión presenta resultados de la investigación Alianzas multisectoriales basadas en estrategias de comunicación, un estudio de caso en el Valle de Aburrá, Colombia, realizada por los investigadores PhD Juan Carlos Molleda, PhD Belio Martínez Jr. y Mg. Ana María Suárez Monsalve, en el proyecto cofinanciado entre la Universidad de Medellín y la Universidad de Florida, 2008-2009. Este análisis se centra en el uso de la estrategia de comunicación como proceso que contribuye con la creación de alianzas multisectoriales. Pretende explicar el proceso de creación de vínculos entre organizaciones representativas de tres sectores: organizaciones comunitarias, empresa privada y Estado. Plantea, como conclusión del análisis, la necesidad de adoptar un rol intermediador por parte del comunicador, evidente en los procesos y productos que genera en la estrategia de comunicación, para hacer coherente el discurso estratégico y la acción comunicativa.
\end{abstract}

Palabras clave: estrategia de comunicación, intermediación social, alianzas.

* Profesora e investigadora en la Facultad de Comunicación, Universidad de Medellín, Colombia. Líder del grupo de investigación Comunicación e Identidad. asuarez@udem.edu.co 


\title{
Alliances based on communication strategies
}

\begin{abstract}
This article presents the interpretation of documents and structured interviews of the investigation "Multi-sector alliances based on communication strategies, a study of case in the Valley of Aburrá, Colombia", realised by PhD Juan Carlos Molleda, PhD Belio Martinez Jr and Mg Ana Maria Suárez Monsalve, in the project co-financed between the University of Medellín and the University of Florida, 2008-2009. This analysis concentrates in the use of the strategy of communication like process that contributes with the creation of multi-sector alliances. It tries to explain the process of creation of bonds between representative organizations of three sectors: communitarian organizations, private company and State. She raises, like conclusion of the analysis, the necessity to adopt a intermediating roll on the part of the signaller, evident in the processes and products that she generates in the communication strategy, to make the strategic speech and the communicative action coherent.
\end{abstract}

Key words: strategy of communication, social intermediation, alliances. 


\section{INVESTIGACIÓN}

Alianzas multisectoriales basadas en estrategias de comunicación participativa, un estudio de caso en el Valle de Aburrá, Colombia, es una investigación realizada en cofinanciación entre la Universidad de Medellín y la Universidad de Florida; está basada en la alianza multisectorial que se creó para la formulación del Plan Estratégico del Norte y el Sur del Valle de Aburrá durante 2005-2007. El objetivo planteado en esta investigación es identificar los elementos que constituyen la estrategia de comunicación efectiva en alianzas multisectoriales que buscan el desarrollo social. Se utilizaron como instrumentos la observación directa, las entrevistas estructuradas, el análisis de contenido de documentos y piezas de comunicación y el grupo focal. Las preguntas de investigación que enmarcan este proyecto son: ¿Una estrategia de comunicación facilita la construcción de alianzas multisectoriales? ¿Cuáles pueden ser los aspectos específicos en el proceso de participación que pueden afectar positiva o negativamente el desarrollo, la implementación y la evaluación de la estrategia de comunicación?

\section{ESTRATEGIA DE COMUNICACIÓN}

En la formulación de la estrategia de comunicación para la alianza multisectorial objeto de investigación, denominada Planes Estratégicos del Norte y el Sur del Valle de Aburrá, se parte del concepto de comunicación ligado a la cultura. En la revisión documental como en los testimonios obtenidos en las entrevistas, se puede constatar que desde un comienzo del proceso de asociatividad se asumió la "comunicación como instrumento para construir cultura", 'según lo expresa en entrevista el coordinador del proceso, "en una alianza los intereses de los actores sí pueden

1 Entrevista semiestructurada realizada en noviembre de 2006, en Sabaneta, Sur del Valle de Aburrá. afectar cuando se entiende la comunicación como difusión o publicidad". Entonces, entender el proceso de comunicación ligado al concepto cultural permitió que se concibieran con intención todos los acercamientos y procesos de interacción como propios de un grupo de individuos que comparten, además de las aspiraciones, construcciones comunes de escenarios y de acciones.

Cuando se analiza el documento formulado para la concepción de la estrategia, se observa que se definieron los principios de comunicación, justificados en que la orientación de los procesos comunicativos requería de una postura filosófica que brindara soporte en valores para la promoción del desarrollo humano y social de los individuos que constituían el público objetivo y el público productor de mensajes. Estos principios fueron: la transparencia, la coherencia, la cohesión y la confianza.

En el mismo documento, se señala la conceptualización que sobre comunicación pública, orientó el proceso de formulación y aplicación de la estrategia. De esta orientación se desprende que el proceso de comunicación tiene un destinatario como componente esencial del mismo y que en la comunicación pública ese destinatario es plural y no singular, por lo que su tratamiento requiere de un amplio conocimiento de las características comunes entre los públicos objetivo. Precisamente, a raíz de esto, la clasificación y caracterización de públicos de la Alianza se convirtió en un proceso vital para comprender las dinámicas para la construcción de sentido colectivo en torno al proceso de la planeación del desarrollo y para involucrar las acciones y transacciones comunicativas que generan relaciones para la concertación.

Las características comunes de los públicos se consideraron para promover la unidad de sentido que contendría la estrategia de comunicación. De esta manera, los públicos pasivos se pudieron convertir en públicos activos, toda 
vez que cobre sentido para ellos el proceso que motiva la alianza.

El tránsito de públicos pasivos a públicos activos se presenta cuando se generan vínculos más que asociativos, vínculos de sentido colectivo (Grunig, 2000). De esta manera, el vínculo con y entre los diferentes públicos fue el principal promotor de la participación y de la apropiación del proceso de la alianza multisectorial. Buscando esto, los grupos de públicos destinatarios de la estrategia de comunicación pública se clasificaron en tres grandes niveles: los pobladores de las zonas norte y sur del Valle de Aburrá, los actores estratégicos (Estado, empresarios, ONG y representantes de organizaciones comunitarias) y legitimadores (políticos, periodistas, líderes gremiales de la región).

Se agrupó a los habitantes o pobladores de la zona, conformados por familias, adultos mayores, adultos, jóvenes y niños, a quienes se llegaría con productos de comunicación de difusión amplia, mensajes de índole informativa y persuasiva, con el fin de lograr en ellos una actitud favorable hacia actividades públicas.

En los actores estratégicos se incluyó a las organizaciones comunitarias de orden artístico, ambiental, cívico, político, de representatividad ciudadana, administradores y funcionarios, consejos municipales de planeación, empresarios de cada municipio y de los diversos sectores de la economía, y medios masivos.

En el nivel de legitimadores se integró a las personas e instituciones que tomaban decisiones administrativas y normativas para asegurar la ejecución y continuidad de los proyectos. Este grupo estaba constituido por la Junta Metropolitana, ${ }^{2}$ los concejos municipales (ediles elegidos por voto popular), las administraciones municipales (alcaldes

2 Conformada por los alcaldes de los municipios que libremente hacen parte del Área Metropolitana del Valle de Aburrá y que preside el Alcalde del Municipio de Medellín. y secretarios) y los organismos de coordinación de los planes estratégicos (consejos directivos y comités técnicos) $)^{3} \mathrm{y}$ en donde se contaba también con líderes de opinión, representantes de los sectores educativo, comunitario y gubernamental.

Al tener identificados los públicos, se especificaron los componentes de la estrategia, como grandes temas que agruparían acciones integradas para lograr efectos sinérgicos. Los componentes definidos fueron: identidad corporativa, información pública, procesos de discusión y concertación y cabildeo.

En el componente identidad visual se concibió el concepto visual que reflejó la alianza y el sentido de los planes estratégicos, utilizando la identidad gráfica en los diferentes escenarios y la generación de piezas de comunicación y publicitarias con la imagen en impresos y elementos digitales, los pendones, la papelería y los productos que se generaron en este componente como logosímbolo, aplicación en pendones, separadores, pasacalles, carátulas y vallas. El diseño de la identidad visual permitió el uso de estos productos en todas las fases del proceso, dándole permanencia al sentido integrador y a la vez independiente de la alianza.

En el componente de información pública se incluyó la gestión informativa dirigida a medios masivos y a los medios locales o comunitarios, con el fin de divulgar los avances e incluir en la agenda pública el proceso generado para la formulación de la alianza, con free press o gestión de la información ante los medios informativos regionales o locales y

3 El Consejo Directivo estaba conformado por el alcalde o su delegado, de cada municipio que intervenía en la alianza, por el director del Área Metropolitana, por los presidentes o directores ejecutivos de las empresas privadas con sede en estas zonas del Valle de Aburrá, por los directores de las organizaciones no gubernamentales que promovieron la Alianza como la Corporación Pro Aburrá Norte, entidad gremial empresarial y por la Cámara de Comercio del Aburrá Sur, la Fundación Social y la Corporación para el Desarrollo de Antioquia. 
la realización de boletín mensual electrónico, página web y productos informativos audiovisuales.

El tercer componente era el proceso de discusión y concertación, donde se concebían todos los espacios del proceso de comunicación para motivar el encuentro voluntario, para escuchar y opinar sobre los documentos y propuestas que realizó el equipo técnico o los consejos establecidos para la participación. Los eventos, talleres, foros y reuniones se constituyeron en espacios de comunicación de doble vía, en los que el intercambio de información, de manera personalizada y directa, consolidó las relaciones, generó confianza y motivó la convocatoria a próximos encuentros de socialización. En este componente se incluyó el diseño y realización de los talleres, la organización y realización de foros abiertos, la orientación de los componentes comunicativos en las actividades de discusión y la realización de material didáctico para los talleres.

Finalmente, el cabildeo o lob6y, concebido como las acciones estratégicas de comunicación para incidir en las decisiones de orden legislativo o ejecutivo, fue realizado con el fin de concertar intereses de las organizaciones involucradas en el proceso de desarrollo. Las acciones de cabildeo estimularon la articulación de procesos institucionales y pusieron de manifiesto el compromiso y la voluntad para la adopción de normas que facilitaran (durante el proceso y en un futuro) la ejecución de los proyectos, producto de la planeación concertada, y las relaciones armónicas entre los grupos interesados. Concebido así, el lobby o cabildeo se concentró en la gestión ante los organismos de representación municipal y metropolitana, la formulación de documentos interinstitucionales, producto de las deliberaciones, y la protocolización de los acuerdos. Esta gestión permitió que las relaciones interinstitucionales se fortalecieran y se concretara en vínculos que promovieran el sentido de lo político en la comunidad del Valle de Aburrá.
Una vez se definieron los componentes de la estrategia comunicacional, se detallaron las tácticas o acciones concretas con las que se aplicaría no sólo el componente sino los indicadores de seguimiento e impacto de cada una de estas acciones.

\section{EVALUACIONES DE LOS ACTORES}

Para esta investigación, otra documentación importante fue la evaluación del proceso, realizado con los representantes de cada uno de los sectores y que señala la valoración de las diferentes estrategias del plan estratégico.

Del análisis de las entrevistas, es importante citar cómo se plantearon momentos clave en el proceso, los mismos que contribuyeron con el éxito la alianza en sus primeras fases. En lo que corresponde a comunicación, la entidad que coordinaba el proceso, en la voz del coordinador, resaltó la conveniencia de haber formulado una estrategia de comunicaciones, y haberla discutido con los actores estratégicos, esto es, con aquellos que conformaron los comités técnicos y los consejos. Si bien se presentó una tensión permanente entre estos actores en relación con el enfoque de la estrategia de comunicación, al final del proceso de formulación del plan se reconoció la validez de la misma y el impacto que generó en los actores sociales representativos de la comunidad que participaron en ella, según testimonio en la misma entrevista.

Entre los aspectos que otros entrevistados destacaron estuvo la concertación y aceptación de una identidad visual propia del proceso que integrara a las diferentes instituciones y organizaciones, tanto públicas como privadas que lideraron la formulación de los planes. Más allá del protagonismo individual de organizaciones públicas o privadas participantes, según los entrevistados, se logró posicionar 
la alianza y el propósito planteado hacia el desarrollo integral.

Además, se destacaron algunos hechos significativos del proceso comunicativo como el posicionamiento de la alianza entre actores participantes. El componente publicitario fue bien enfocado y producido, las piezas garantizaban la identidad a través de la imagen y el diseño, además de mantener el criterio de la publicidad al servicio de la acción comunicativa.

De igual manera, algunos entrevistados resaltaron la publicación en medios masivos de los avances de la alianza, destacándola como noticia para los medios de comunicación en los momentos clave del proceso; con un manejo acertado de relaciones públicas y fomentando el interés en los medios locales, "había que competir con otras noticias en medio del sensacionalismo que vivimos y con los medios locales, periódicos, emisoras". Desde esta perspectiva, también se obtuvo, como logro, la documentación del proceso de manera acertada y con calidad informativa y de diseño visual.

El grupo focal reconoció el enfoque comunicativo que procuraba, más allá de la figuración de entidades y personas, el acto comunicativo para incentivar la comprensión del proceso, la confianza y la participación en el mismo. Como lo planteó uno de los participantes en este grupo: "La mayoría de la población es conocedora de que existe un plan que los está orientando a nivel de su región, y eso no se hubiera dado, ese conocimiento, ese plan no se hubiera dado si los hubieran diseñado simplemente x o y actor en una oficina... pero eso se dio a conocer rápidamente por la participación activa de diferentes actores" .

En cuanto a los documentos que recogen la memoria del proceso, se consigna la realización

4 Grupo Focal realizado con 10 actores representantes de diferentes sectores, en agosto de 2008 en el Girardota, municipio del norte del Valle de Aburrá. de 5 actividades con los diferentes grupos que participaron en los planes estratégicos: Comité Técnico Norte, Comité Técnico Sur, Delegados Norte, Delegados Sur y una evaluación conjunta del Consejo Asesor. Participaron 73 actores, representantes de los diferentes sectores y cada uno realizó la evaluación y después se discutió en plenaria.

El Comité Asesor y el Comité Técnico del Norte destacaron la Estrategia de Comunicación como una de las razones por las cuales se obtuvieron logros significativos como el reconocimiento de la zona como unidad, la participación de actores, identificar y formular visión conjunta, la concertación interinstitucional, la generación de confianza entre los actores.

Aún así, en la evaluación de los delegados de la comunidad del Norte, se señala como una de las dificultades del proceso el no haber hecho más divulgación, lo que condujo a menor participación de la comunidad. También lo señalan los delegados del Sur diciendo: "faltó mayor difusión del plan". ${ }^{5}$

Luego, la evaluación deja valoraciones significativas del proceso estratégico de la comunicación, planteado en los documentos y ejecutado en el transcurso de implementación de la Alianza. Sin embargo, se destaca la insatisfacción por la divulgación en la comunidad, en parte, porque el proceso representativo hace más dispendiosa la entrega de información, aunque la participación se logra a partir del conocimiento de lo que la Alianza se trazó como objetivo: la planificación de la región.

\section{EL DESARROLLO INTEGRAL}

Con el análisis de entrevistas, grupo focal y análisis documental, se observa una constante

5 Análisis documental realizado en mayo de 2009, con la información obtenida de los archivos y sistematización del proceso de formulación de los Planes Estratégicos del Norte y Sur del Valle de Aburrá 
en la valoración de esta alianza multisectorial. Sin duda, en los testimonios y en las evaluaciones, sale a relucir el papel de la entidad coordinadora del proceso para la formulación de los Planes Estratégicos del Norte y Sur del Valle de Aburrá. La orientación de esta Organización No Gubernamental es coherente con la formulación institucional de un modelo para promover el desarrollo integral de las comunidades.

El modelo DIL, Desarrollo Integral Local, construido por la Fundación Social, entidad coordinadora de la Alianza, se fundamenta en unos principios y en una propuesta de planeación y gestión participativa del desarrollo local, entendiendo que "El desarrollo tiene una dimensión espacial y una temporal. La especialidad del desarrollo, en nuestra época, plantea la continuidad entre lo local y lo global, y se expresa en un proceso de urbanización de la vida que involucra lo rural, y lo pone a su servicio. La localización tiene una importancia central pero siempre atravesada, resignificada por la dinámica de la globalización" (Quintero, 2004, p. 26). Este modelo ha sido evaluado y reevaluado por esta organización durante décadas y con experiencia en diferentes regiones del país, pero lo que interesa resaltar es que en este modelo siempre ha estado presente la comunicación como componente fundamental para el desarrollo. En el texto ya citado, las características generales de las dimensiones básicas del desarrollo, sobre las cuales se enfoca la organización, plantean que "El desarrollo integral, como un proyecto común, sólo será posible con la participación de todos los actores estratégicos y por tanto, requiere que se visualice, se sueñe, se ponga al alcance de todos. Es necesaria la concertación de sentidos compartidos, de significaciones comunes, de imaginarios colectivos favorables al desarrollo deseado para hacerlo comprensible en forma similar y accesible a todos". ${ }^{6}$ Este planteamiento orienta el componente comunicacional

6 Fundación Social (2004) Serie DIL, Desarrollo Integral Local. Bogotá. del desarrollo integral local y que se concreta, luego, como uno de los elementos de las líneas de actuación del modelo. Esa línea de actuación es la construcción del sentido de lo público y en este, la comunicación para el desarrollo y la paz.

La Declaración del Milenio (Naciones Unidas, 2000) centró las prioridades para alcanzar el desarrollo equitativo y justo del mundo. Hizo un llamado para la erradicación de la pobreza, la protección del entorno, en los derechos humanos, la democracia y el buen gobierno, y para la protección de las personas vulnerables. Aún hoy, casi una década después, estas son las ideas que deben centrar, en acuerdo para el mundo, el trabajo presente y futuro, para superar la inequidad, la exclusión y todas las consecuencias de un pensamiento y una acción desenfrenados por el supuesto progreso que nos gobernó durante el siglo pasado. La evaluación en estos 9 años nos muestra cómo las palabras o las declaraciones, sin acción y decisión, no conducen al cambio. Aún estamos a tiempo de movilizar la acción social, económica y política para generar un mejor vivir en los tiempos actuales y por venir. La comunicación y las relaciones públicas deben estar a ese servicio.

La discusión mundial sobre el desarrollo ha dicho ya que éste ha de ser humano, integral, sostenible y participativo; estas características nos remiten a la condición humana centrada en las personas y potenciando sus posibilidades de realización, incluyendo los ámbitos educativos, familiares, laborales y la utilización del tiempo libre; también se focaliza en la condición integral pues ya se ha cometido el error de exaltar el carácter económico del desarrollo. Se hace urgente concretar el desarrollo en la dimensión política, cultural y ambiental de la sociedad.

Los desafíos sociales, económicos, políticos, y ambientales de la sociedad en todo el mundo están exigiendo la intervención y la colaboración 
entre el sector gubernamental, el sector privado, el sector no lucrativo, el sector comunitario y las organizaciones cívicas. Lamentablemente son muchos los ejemplos de los panoramas complejos que se viven en el mundo, los que han requerido recursos de diferentes sectores y la intervención masiva y prolongada de gobiernos, comunidad y empresa privada para salir de las crisis. Las organizaciones, independientemente del sector al que pertenezcan aportan y combinan recursos, conocimiento y experiencia que, a la vez, crean sociedades entre diferentes sectores, buscando contribuir con la superación de los problemas, y al final, promueven el cambio social, el desarrollo económico y la unión de nación/comunidad.

La responsabilidad social corporativa, la filantropía, el planeamiento estratégico y el desarrollo sustentable son factores que influencian esta dinámica social.

\section{COMUNICACIÓN Y RELACIONES PÜBLICAS PARA LA INTERMEDIACIÓN SOCIAL}

Los desafíos sociales, económicos, políticos, y ambientales de la sociedad en todo el mundo están exigiendo la intervención y la colaboración entre el sector gubernamental, el sector privado, el sector no lucrativo, el sector comunitario y de organizaciones cívicas. Diferentes organizaciones aportan y combinan recursos y experiencia para crear sinergia y promover sociedades multisectoriales que orienten el cambio social, el desarrollo económico, y la unión de nación/comunidad. Su actuación y su misión identifican necesidades y soluciones para atender los problemas que afectan a las organizaciones mismas y a la alianza en su totalidad.

Alcanzar y mantener acuerdos en medio del desafío que implican estas sociedades multisectoras se apoya, a menudo, en profesionales de comunicación y relaciones públicas que puedan aceptar una responsabilidad extendida: actuar como mediadores. Esta visión se ha hecho presente con la perspectiva social de relaciones públicas. Desde este punto de vista, y con el análisis de diferentes experiencias y orientaciones, se hace indispensable reconocer que la comunidad valora las experiencias de sus miembros, y por tanto, es necesario poner esa experiencia en común, trabajar conjuntamente hacia metas comunes y facilitar la participación de los diversos grupos para que la experiencia, las habilidades, y los recursos resulten en soluciones más amplias y mejoren la calidad de vida. Teniendo como referencia a diferentes autores (Hallahan, 2004, Kruckeberg y Starck, 1988; Martinez y Kiousis, 2006; Starck y Kruckeberg, 2001, 2003), Molleda (2001) explica que una perspectiva latinoamericana, convoca a los profesionales de las relaciones públicas para ser agentes de la "transformación social" y parte de la "conciencia social" de sus organizaciones. Además, las relaciones públicas en América Latina apuntan a contribuir al bienestar de los ambientes sociales donde las organizaciones funcionan.

También Suárez (2003) había planteado la contribución del comunicador en la transformación social: "las acciones de comunicación en las organizaciones no pueden pensarse fuera del efecto que generan en la sociedad. De hecho, en cada empresa pública o privada, se constituyen sociedades que interactúan por largos períodos y luego en otros espacios como los familiares, barriales, gremiales, expresan aquellos procesos informativos y comunicativos de que fueron objeto en el lugar de trabajo" y esto se basa también en la consideración de que la comunicación tiene una perspectiva educativa en las organizaciones.

De igual manera Suárez (2008) plantea, a propósito de la reflexión sobre ese papel social expresado en el diseño de estrategias de comunicación para la construcción de identidad que 
Cuando se define una estrategia de comunicación, uno de los primeros actos de concertación es la definición del propósito o propósitos a alcanzar fruto de esa acción estratégica. Para que lo sea, es decir, para que sea verdaderamente estratégica, es necesario considerar una serie de variables que están marcando el contexto de la acción comunicativa. Desde una posición responsable del equipo de comunicaciones, es prioritario establecer los valores o principios que orientarán la acción estratégica, y por supuesto, las tácticas tendrán que responder a esta orientación. Para ponerlo en otro lenguaje, cada producto de comunicación, llámese pieza publicitaria, evento, medio informativo, no existe por sí sólo en la interacción organizativa, corresponde con un propósito y se espera de él un efecto en el comportamiento de los públicos a quienes van dirigidos. Resulta que en la acción cotidiana es difícil observar espacios propicios para inducir un cambio en la mentalidad directiva para ajustar los discursos a la coherencia entre el decir y el hacer de las empresas. Sin embargo, desde una postura crítica de la comunicación, tiene que estar presente el interrogante en el equipo profesional de comunicación, la pregunta fundamental por cómo construir identidad con las interacciones que promuevo, como profesional, a través de productos audiovisuales y gráficos, y de procesos corporativos que motivan la interacción social (Suárez, 2008).

En este contexto, los esfuerzos de un profesional de las relaciones públicas son tan buenos como las herramientas estratégicas que utiliza. Precisamente, para contribuir con la formación de los comunicadores y relacionistas, el propósito de este estudio es ilustrar cómo la comunicación participativa es un enfoque fundamental para los profesionales de las relaciones públicas que apoyan esfuerzos de diferentes sectores de la sociedad.
Como en muchos otros países en vías de desarrollo, los profesionales de las relaciones públicas en Colombia desempeñan un papel social en la transformación dentro de su entorno social (Molleda y Suárez, 2006): "el ambiente político y económico volátil de hoy en Colombia, demanda que los profesionales de las relaciones públicas se centren en la necesidad de desarrollar campañas para construir confianza en sus organizaciones, para buscar la paz y para promover cambios en la sociedad como esfuerzos para construir nación" (p. 28).

La noción de comunicación estratégica se utiliza en esta investigación para referirse a la realización de actividades interinstitucionales que tienen como objetivo dedicarse a los principales grupos de interés, internos y externos, para lograr las metas y los objetivos compartidos. La comunicación desde una perspectiva participativa provee a los miembros de una sociedad los mecanismos de comunicación necesarios para intervenir en la toma de decisiones. Por lo tanto, las alianzas multisectoriales, según una perspectiva estratégica, son el producto de la investigación eficaz, la introspección, la transparencia, la interacción y la creatividad. Estas dos ideas se unieron para denotar esos esfuerzos interorganizacionales que son el resultado directo, y el catalizador para la concertación entre los socios respecto de un tema o una situación, para formar alianzas con el fin de alcanzar objetivos estratégicos.

\section{ANÁLISIS}

En un primer artículo publicado sobre esta investigación Molleda, Martínez y Suárez (2008) plantean, citando a Enz, Inman, y Lankau (2000): "desde la revolución industrial, el diseño dominante para las alianzas alrededor del mundo se ha basado en la teoría económica, en la justificación de los intereses particulares y la riqueza" (p. 185). A pesar de su foco en el desarrollo sostenible 
promovido por corporaciones multinacionales, también amplían el alcance de sociedades para incluir ciudadanos, agencias públicas, negocios locales y gobierno.

Las alianzas multisectoriales están cambiando la forma y la permanencia de sociedades, porque los miembros tienen diferentes maneras de operar y actuar. Por esto las alianzas multisectoriales deben adoptar, en su totalidad, un sistema común de metas y de valores.

Para esta investigación, se tomó como base la clasificación que Austin, Reficco, y el equipo de investigación de SEKN (2004a), quienes resumen tres fases de colaboración para el desarrollo de alianzas: filantrópico, transaccional e integrativo. La etapa filantrópica exige las donaciones corporativas que atienden a peticiones de nonprofits. La transaccional es la etapa de actividades específicas entre las organizaciones privadas y no lucrativas con un intercambio de doble vía. Por último, la etapa integrativa incluye las "alianzas estratégicas que implican encarar misiones, sincronizar estrategias y la compatibilidad de valores" (Austin et al., 2004, p. 5). Austin (2000) enfatiza que las "relaciones de colaboración emigrarán cada vez más del tradicional foco filantrópico, caracterizado por el donante benévolo y el receptor agradecido, hacia alianzas más profundas, estratégicas" (p. 1). De acuerdo con el análisis y basados en esta clasificación, el equipo de investigación concluyó que la alianza multisectorial documentada y analizada en este estudio siguió el componente integrativo en el cual la comunicación y las relaciones públicas fueron utilizadas para ayudar a alcanzar más profundidad en la alianza, coordinada para el logro de metas comunes y ventajas sociales equitativas para los actores que intervinieron en ella.

De otro lado, Austin et al. (2004b) explica cinco dimensiones principales del proceso de alianza. La construcción de los puentes intersectoriales es la primera dimensión, que implica identificar los motivos que tienen cada sector y cada organización en colaborar, superando barreras. Para ello buscan un interlocutor o un portavoz, capitalizan las diversas capacidades institucionales preexistentes, motivan el reconocimiento de la cultura de cada organización y valoran la comunicación eficaz. La segunda dimensión es la alineación del acuerdo: articular un sistema compartido de expectativas de "las cuales cada socio individualmente examina cómo esta colaboración potencial cabe en su realidad de organización" (Austin et al., 2004c, p. 75). La generación del valor para las compañías y para las comunidades combinando los recursos dominantes es la tercera dimensión (Austin et al., 2004d). La cuarta dimensión maneja la relacióninstitucionalización de la sociedad dentro de organizaciones miembro de la alianza y de sus stakeholders. También exige promover el papel de comunicaciones internas y externas, y los recursos para la construcción de confianza entre socios (Austin et al., 2004e). Finalmente, la quinta dimensión, crecimiento e innovación, consiste en aprendizajes y conocimientos compartidos para mejorar las intervenciones futuras (Austin et al., 2004f). La literatura sobre la construcción de alianzas explora el papel de la comunicación en términos generales. Este estudio procura hacer una contribución identificando el papel de la comunicación y las relaciones públicas en la creación y el manejo de sociedades multisectoriales.

\section{CONCLUSIONES}

Los resultados del análisis de esta experiencia indican que las alianzas requieren una expresión muy clara de los propósitos colectivos; también, que es necesario evitar la dominación de los actores que en ella intervienen, identificar metas compartidas y mensajes clave que fortalezcan la confianza de los diferentes sectores, además de 
un proceso dialógico transparente, inclusivo y permanente para alcanzar la sinergia.

Los comunicadores y relacionistas podemos actuar como intermediarios sociales. Identificar los intereses de diferentes actores en una alianza multisectorial, respetarlos y ponerlos en sintonía requiere la habilidad de reconocer la naturaleza humana y la naturaleza de las organizaciones. Más allá de tomar partido es necesario buscar el equilibrio entre los intereses y, bajo la premisa de compartir responsabilidades y logros, identificar lo común: el trabajo por el desarrollo humano. La tradicional operatividad de los profesionales de la comunicación está reemplazándose, por fortuna, por la comunicación y las relaciones públicas que potencian la acción transformadora de la comunicación en la sociedad y eleva su estatus en beneficio de los comunicadores y de la comunidad en general.

El ejercicio profesional se integra a la realidad social del contexto local por medio de intervenciones como esta experiencia, en la que la investigación y la docencia se ponen al servicio de la gestión social. La vinculación entre Administración Pública, empresa, universidad y comunidad, intermediada por la comunicación y las relaciones públicas, es un reto que no debe ser ocasional, sino constante en el contexto latinoamericano. Para lograr alcanzar este reto es importante tener un modelo, que, como bien se ha planteado en Colombia, promueva un tipo de comunicación que contribuya con el desarrollo social, con procesos de diálogo y cooperación (Red de Gestores Sociales, 2006).

Analizar, registrar y divulgar este tipo de proyectos es una oportunidad para quienes en el día a día se enfrentan con el reto de plantear alternativas que conjuguen diversas variables como tiempo (urgencia), múltiples intereses (conflictos), confianza (todos por un mismo propósito) trascendencia (desarrollo humano).
Con participación en la gestión del desarrollo social, los comunicadores y relacionistas estamos respondiendo a esa solicitud por construir un lenguaje común para el entendimiento social y estamos trabajando para generar acciones de comunicación que permitan reconocer cómo es la sociedad de nuestro país (Correa, E. Galvis, C.A E Suárez, A.M 2006), por eso nos atrevemos a plantear que sí existe en el ámbito de lo público y de lo privado la conciencia en la necesidad de un comunicador y relacionista, que no sólo registre hechos comunicativos, sino que los propicie, los promueva y los ejecute como mediador social, y los administre teniendo en cuenta los intereses de los diferentes actores de una sociedad.

El compromiso con el desarrollo humano, integral y sostenible, incluye a comunicadores que se desempeñen en cualquier campo de actuación de la profesión. La tendencia al utilitarismo de los medios, de la publicidad y la imagen corporativa es revaluada por la demanda que hacen nuestros pueblos para unir voluntades en torno a un desarrollo armónico, equitativo y digno.

Cualquier proceso o gestión social requiere de la comunicación, de sus estrategias y tácticas, pensadas para lograr consensos y orientar el disenso a posibilidades no violentas. Por lo tanto, el profesional tiene que saber diseñar estrategias de comunicación y relaciones públicas que soporten el peso de múltiples intereses y que orienten la gestión hacia resultados tangibles en la calidad de vida de los públicos destinatarios e involucrados en estos procesos.

La comunicación, sus medios y procesos, al servicio del desarrollo, trasciende la función informativa cuando, efectivamente, la acción comunicativa se dirige a públicos específicos para generar acciones y comportamientos que los involucren en procesos sociales. 


\section{REFERENCIAS BIBLIOGRÁFICAS}

Alianza estratégica para el desarrollo del Aburrá Sur. 2006. Planes Estratégicos Norte y Sur del Valle de Aburrá. Fundación Social, regional Medellín: Medellín.

Fundación Social. 2004. Serie DIL, Desarrollo Integral Local. Bogotá.

Suárez, Ana María. 2008. "Intervención del comunicador en la construcción de identidades". En: Galvis, C E Suárez, A. M (editores académicos). Investigación en comunicación, vigencia y prospective. Comunicación pública E Comunicación e Identidad: Medellín: Sello Editorial Universidad de Medellín.

Grunig, James. 2000 Dirección de Relaciones Públicas. Barcelona: McGraw Hill.

Hallahan, K. 2004. "Community" as a foundation for public relations theory and practice. Communication Yearbook, 28, 233-279.

Kruckeberg, D., \& Starck, K. 1988. Public relations and community: A reconstructed theory. New York: Praeger.

Molleda, J. C., \& Suárez, A. M. 2006. Factor analysis to identify the social roles of Colombian public relations professionals. Glossa, 1(1). Available at http://bibliotecavirtualut.suagm.edu/Glossa/Journal/Glossa.htm

Molleda, J. C. \& Suárez, A. M. 2003 Julio-diciembre. "The Role of Colombian Public Relations Professionals as Agents of Social Transformation". Revista Anagramas, rumbos y sentidos de la comunicación No. 3, 87. Medellín: Sello Editorial Universidad de Medellín.

Molleda, JC, Martínez Jr, B \& Suárez, AM (2008 Enero-Junio) Building Multi-sector partnerships for progress with strategic, participatory communication: a case study from Colombia. Revista Anagramas, rumbos y sentidos de la comunicación No. 12, 105. Medellín: Sello Editorial Universidad de Medellín.

Red de Gestores Sociales, Consejería Presidencial de Programas Especiales y Ministerio de Comunicaciones de la Presidencia de la República de Colombia. (2005). Guía de comunicación para la gestión y el desarrollo social.

Sandoval, Carlos. 1997. Investigación cualitativa. ICFES, Santafé de Bogotá.

Starck, K., E Kruckeberg, D. 2003. Ethical obligations of public relations in an era of globalisation. Journal of Communication Management, 8(1), 29-40.

Suárez, A. M. 2003 Enero-junio. Responsabilidad social de la comunicación corporativa. Revista Anagramas, rumbos y sentidos de la comunicación No. 2. Medellín: Sello Editorial Universidad de Medellín. 